\title{
Very early onset inflammatory bowel disease in a South Asian country where inflammatory bowel disease is emerging: a distinct clinical phenotype from later onset disease
}

\author{
Rupa Banerjee, Partha Pal, Zaheer Nabi, Upender Shava, Girish Ganesh, D. Nageshwar Reddy \\ Asian Institute of Gastroenterology, Hyderabad, India
}

Background/Aims: Information on pediatric inflammatory bowel disease (PIBD) and very early onset IBD (VEOIBD) are sparse in India, where IBD is emerging. We aimed to evaluate characteristics of VEOIBD and later onset PIBD (LO-PIBD) in India. Methods: We performed retrospective analysis of a large, prospectively maintained IBD registry. PIBD was divided in to VEOIBD ( $<6$ years) and LO-PIBD (6-17 years). Demographic data, disease characteristics and treatment were compared between the PIBD groups and with other Asian/Western studies as well as the adult patients of the registry. Results: Of 3,752 IBD patients, 292 (7.8\%) had PIBD (0-17 years) (175 Crohn's disease [CD], 113 ulcerative colitis [UC], 4 IBD-undifferentiated; 22 VEOIBD [7.5\%], and 270 LO-PIBD [92.5\%]). VEOIBD patients had more severe disease compared to LO-PIBD in both UC $(P=0.003)$ and CD $(P<0.001)$. Familial IBD was more common in VEOIBD $(13.6 \%)$ compared to LO-PIBD $(9.2 \%)$. Ileal disease (L1) was an independent risk factor for diagnostic delay in pediatric CD. Diagnostic delay ( $>6$ months) was significantly lower in VEOIBD $(40.9 \%)$ than in LO-PIBD $(78.8 \%)(P<0.001)$. Compared to other Asian and Western studies, extensive UC $(72.5 \%)$ and complicated CD (stricturing/penetrating: $42.7 \%$ ) were relatively more common. Perianal CD was relatively less frequent (7.4\%). PIBD had a significantly higher number of complicated and ileal CD and extensive UC comparison to adult cohort of the registry. Conclusions: VEOIBD has more aggressive phenotype than LO-PIBD. Disease appears distinct from other Asian and Western studies and adult onset disease, with more complicated CD and extensive UC. (Intest Res 2021;19:398-407)

Key Words: Inflammatory bowel diseases; Crohn disease; Ulcerative colitis; Very early onset IBD; Later onset pediatric IBD

\section{INTRODUCTION}

Pediatric inflammatory bowel disease (PIBD; age $<17$ years) constitutes up to $10 \%-25 \%$ of all IBD patients. ${ }^{1}$ Of these, a small proportion are very early onset IBD (VEOIBD; <6 years). VEOIBD appears to be a distinct subset of pediatric IBD with unique epidemiological and phenotypic characteristics in-

Received August 24, 2020. Revised October 13, 2020.

Accepted October 17, 2020.

Correspondence to Rupa Banerjee, Asian Institute of Gastroenterology, 1 ,

Mindspace Rd, Gachibowli, Hyderabad, Telangana 500032, India. Tel: +91-

9849287530, Fax: +91-40-23324255, E-mail:dr_rupa_banerjee@hotmail.

com cluding a severe and aggressive course of disease, predominant colonic involvement and a higher incidence of familial IBD compared to PIBD other than VEOIBD (6-17 years). ${ }^{1}$

The incidence of PIBD is on the rise globally. ${ }^{2}$ A complex interplay of genetic and changing environmental factors has been implicated for the rising incidence although specific factors are yet to be completely understood. ${ }^{2-6}$

Most of the epidemiological data however are from the West including North America, Canada, and Europe. ${ }^{3}$ A few recent studies from Asia also suggest a rising trend of PIBD. Interestingly the phenotype appears to be distinct from that of the West including a milder ulcerative colitis (UC) and a higher perianal 
involvement in Crohn's disease (CD) ${ }^{4-10}$ India is a large country with a large population where IBD is a new but emerging disease. Though the incidence of IBD is lower than that of the West expectedly, the burden of disease is high. The diagnosis of IBD itself poses significant challenges in a country where infectious diseases predominate, and tuberculosis (TB) is endemic. $^{11,12}$

Data on PIBD behavior and outcomes from India is limited. There are no published reports or separate analysis of VEOI$\mathrm{BD}$ and its differences from later onset disease. Similarities to adult IBD and distinctive features have been described in small and questionnaire-based studies. ${ }^{5,13-20}$

The primary aim of this study was to evaluate the disease characteristics and treatment patterns in VEOIBD and compare that to later onset PIBD (LO-PIBD; 6-17 years). We also compared our data with other PIBD or VEOIBD studies from Asia and the West.

\section{METHODS}

\section{Study Population}

The study was conducted at the Asian Institute of Gastroenterology (AIG) in Hyderabad, India, a gastroenterology tertiary center for IBD patients across India. The IBD center was established in 2004 with a well-established prospectively maintained registry. ${ }^{21}$

The registry includes the detailed history and demographic profile since the first visit, symptoms, disease activity scores, medication, and family history collected by interview and chart review. Clinical characteristics, including family history and response to treatment, are updated at each follow-up visit. ${ }^{12,22}$

Eligible subjects included all patients with confirmed IBD who were less than 17 years of age up to November 2019. All patients underwent at least one ileo-colonoscopy and gastroscopy before enrolment into the registry. Patients with a followup of less than 6 months were excluded from the study.

The diagnosis of IBD was based on the European Society for Pediatric Gastroenterology Hepatology and Nutrition (ESPGHAN) "Porto" criteria including history, clinical, endoscopic, histologic and radiologic features. ${ }^{23}$ Small bowel evaluation was done by magnetic resonance enterography in majority of the cases. Computed tomography enterography was used in very limited numbers due to risk of radiation. A chest X-ray, 2 step Mantoux and biopsy specimen polymerase chain reaction for Mycobacterium. TB DNA were done to rule out TB in CD patients. Clinical, endoscopic, radiologic features and his- topathology were considered for differentiating intestinal $\mathrm{TB}$ from CD. Empirical anti-TB therapy (ATT) was initiated when diagnostic dilemma persisted even after extensive evaluation. An assessment for response is done at 2 months. In case of improvement, ATT is continued for at least 6 months.

The patients were classified as VEOIBD $<6$ years of age, and LO-PIBD for patients between 6 and 17 years of age. The subgroup of patients less than 2 years of age were identified as infantile onset IBD (IOIBD). Demographic data including age, sex, family history, clinical symptoms at presentation (abdominal pain, diarrhea, weight loss, and rectal bleeding) and presence of extraintestinal manifestations was retrieved from the registry. The diagnostic delay was calculated based on the difference between the age of onset of symptoms and the age at diagnosis.

\section{Disease Extension and Severity}

The location of the disease was noted using the pediatric modification of Montreal classification (Paris classification) for the purpose of the study. ${ }^{24}$ The disease activity at presentation was recorded according to the Pediatric Crohn's Disease Activity Index (PCDAI) and the Pediatric Ulcerative Colitis Activity In$\operatorname{dex}$ (PUCAI). ${ }^{25,26}$ The maximum extent recorded and the maximum severity noted were included in the analysis.

The treatment given including the requirement of steroids, immunomodulators and biologics was presence of complications and need for surgeries were recorded.

\section{Statistical Methods}

The IBD registry runs on Apex healthcare management system software which allows entry of follow-up data at each visit. The data for the PIBD and VEOIBD patients were reviewed for completeness and consistency before entering into Microsoft Excel (Microsoft Corp., Redmond, WA, USA).

Continuous variables were expressed as mean $(95 \%$ confidence interval $[\mathrm{CI}]$ ) if normally distributed or as median and interquartile range (IQR)/range if not normally distributed. Normality of distribution was ascertained by the KolmogorovSmirnov test and the Shapiro-Wilk test $(P<0.05$ indicates nonnormal distribution). Categorical variables were expressed as frequencies and percentages. Patient characteristics were compared using Student $t$-test (for data with normal distribution) and Mann-Whitney test (for non-parametric data/data with non-normal distribution with 2 groups) for continuous variables and chi-square/Fisher exact test (depending on sample size and expected values) for categorical variables. Univariate 
followed by multivariate logistic regression analysis was performed to look for risk factors of diagnostic delay ( $>50$ th percentile) in CD and UC and also to assess risk factors for surgery in CD. $P$-values of $<0.05$ were considered statistically significant. The data were analyzed using SPSS version 21.0 (IBM Corp., Armonk, NY, USA).

\section{Ethical Considerations}

All patients seen at the clinic with a confirmed diagnosis of IBD are enrolled into the registry after informed consent including permission to use information for research purposes. For pediatric cases (age of onset $<17$ years) informed consent is obtained from the parent or legal guardian. The study was approved by the Institutional Review Board of Asian Institute of Gastroenterology (AIG AHF IRB No. 07/2019) and performed in accordance with the principles of the Declaration of Helsinki.

\section{RESULTS}

\section{Demographics and Disease Presentation}

The AIG IBD registry included 3,752 patients. Of these, 292 subjects (7.8\%) comprised the PIBD cohort (age < 17 years). Majority ( $\mathrm{n}=270,92.5 \%)$ of the PIBD cases belonged to the LO-PIBD group ( $6-17$ years). VEOIBD ( $<6$ years) and IOIBD ( $<2$ years) were identified in $22(7.5 \%)$ and $8(2.7 \%)$ children, respectively. The distribution of the pediatric cases according to age is shown in Fig. 1A.

There were more cases of CD $(59.9 \%, 175 / 292)$ than UC $(38.7 \%, 113 / 292)$ in all the age groups. IBD-undifferentiated (IBD-U) was seen in 4 patients (1.4\%). CD was diagnosed in $13(59.1 \%)$ and UC in 8 (36.3\%) of VEOIBD cases. IBD-U was seen in 1 patient (4.5\%) of VEOIBD group.

The presenting symptoms of VEOIBD are tabulated in Tables 1 and 2. There were no significant differences in the
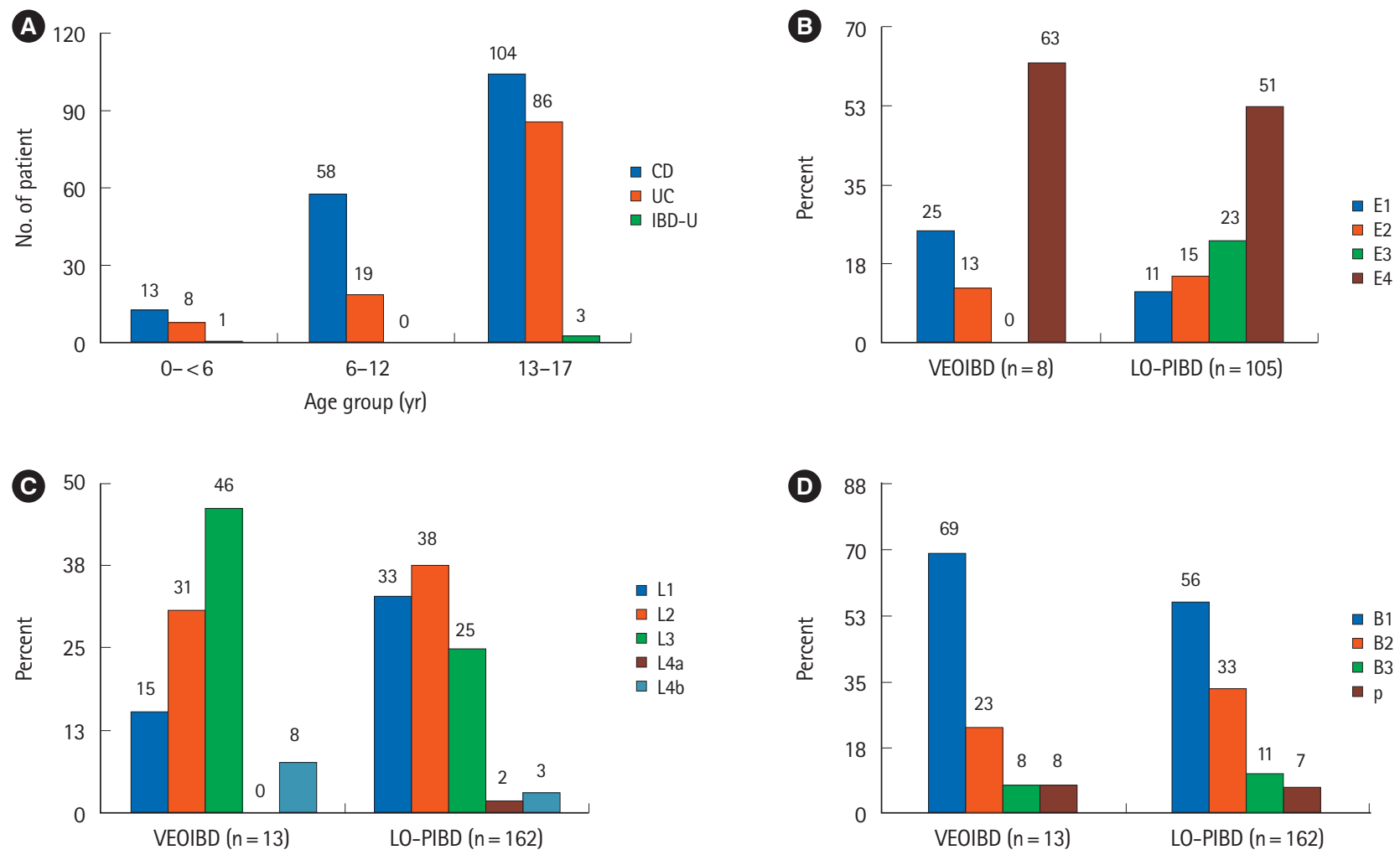

Fig. 1. Age distribution, disease extent, and phenotype of PIBD. (A) Distribution of $U C$ and $C D$ cases according to age groups. (B) Extent of disease in UC: VEOIBD and LO-PIBD patients. (C) Extent of disease in CD: VEOIBD and LO-PIBD patients. (D) Disease behavior in CD: VEOIBD and LO-PIBD patients. IBD, inflammatory bowel disease; CD, Crohn's disease; UC, ulcerative colitis; IBD-U, undifferentiated IBD; VEOI$B D$, very early onset IBD; LO-PIBD, later onset pediatric IBD; E1, proctitis; E2, upto splenic flexure; E3, upto hepatic flexure; E4, pancolitis; L1, ileal; L2, colonic; L3, ileo-colonic; L4a, upper gastrointestinal disease upto ligament of Treitz; L4b, beyond ligament of Treitz upto distal ileum $C D ; B 1$, inflammatory; $B 2$, stricturing disease; $B 3$, fistulizing $C D ; p$, perianal disease. 
Table 1. Comparison of VEOIBD ( $<6$ Years) and LO-PIBD (6-17 Years)

\begin{tabular}{|c|c|c|c|}
\hline Variable & $\begin{array}{l}\text { VEOIBD, } \\
\text { No. (\%) }\end{array}$ & $\begin{array}{c}\text { LO-PIBD, } \\
\text { No. (\%) }\end{array}$ & $P$-value \\
\hline No. of patients & $22(7.5)$ & $270(92.5)$ & \\
\hline Ratio of CD:UC & $1.62: 1$ & $1.55: 1$ & \\
\hline Age of onset of symptom (yr) & & & $<0.001$ \\
\hline Mean \pm SD & $3.11 \pm 1.49$ & $13.91 \pm 2.97$ & \\
\hline Median (IOR) & $3(2-4)$ & $15(12-16)$ & \\
\hline Male sex & $18(82)$ & $165(61)$ & 0.053 \\
\hline \multicolumn{4}{|l|}{ Diagnostic delay } \\
\hline Median (IOR) (mo) & $6(2-30)$ & $12(7-24)$ & 0.034 \\
\hline Diagnostic delay > 6 mo & $9(40.9)$ & $213(78.8)$ & $<0.001$ \\
\hline \multicolumn{4}{|l|}{ Symptoms } \\
\hline Diarrhea & $17(77.2)$ & $184(68.1)$ & 0.370 \\
\hline Blood in stool & $15(68.2)$ & $142(52.6)$ & 0.160 \\
\hline Mucus in stool & $11(50.0)$ & $91(33.7)$ & 0.120 \\
\hline Weight loss & $8(36.4)$ & $136(50.4)$ & 0.210 \\
\hline Abdominal pain & $11(50.0)$ & $181(67.0)$ & 0.110 \\
\hline Fever & $8(36.4)$ & $78(28.9)$ & 0.450 \\
\hline No. of patients with EIMs & $5(22.7)$ & $59(21.8)$ & 0.550 \\
\hline Uveitis & $1(4.5)$ & $8(2.9)$ & \\
\hline Skin & $2(9.1)$ & $25(9.2)$ & \\
\hline Arthritis & $3(13.6)$ & $40(14.8)$ & \\
\hline PSC & 0 & $2(0.7)$ & \\
\hline Family history of IBD & $3(13.6)$ & $25(9.2)$ & 0.700 \\
\hline History of consanguinity & $3(13.6)$ & $18(6.6)$ & 0.380 \\
\hline History of appendicitis & 0 & $2(0.7)$ & - \\
\hline \multicolumn{4}{|l|}{ Treatment received } \\
\hline 5 -ASA & $20(90.9)$ & $261(96.7)$ & 0.190 \\
\hline Use of steroids & $14(63.6)$ & $152(56.3)$ & 0.500 \\
\hline Immunomodulators & $8(36.4)$ & $68(25.2)$ & 0.250 \\
\hline Use of biologics & $2(9.1)$ & $15(5.5)$ & 0.630 \\
\hline AT & $1(4.5)$ & $32(11.8)$ & 0.490 \\
\hline History of surgery & $3(13.6)$ & $29(10.7)$ & 0.720 \\
\hline
\end{tabular}

$I B D$, inflammatory bowel disease; VEOIBD, very early onset IBD; LO-PIBD, later onset pediatric IBD; $C D$, Crohn's disease; $U C$, ulcerative colitis; $S D$, standard deviation; IOR, interquartile range; EIM, extraintestinal manifestation; PSC, primary sclerosing cholangitis; ASA, aminosalicylic acid: $\mathrm{A} T$, anti-tubercular therapy.

symptoms at onset, presence of extraintestinal manifestations and treatment received between VEOIBD and LO-PIBD (Tables $1,2)$.

\section{Family History}

A family history of IBD was noted in 3 (13.6\%) of VEOIBD and $25(9.2 \%)$ children with LO-PIBD $(P=0.70)$. Three $(13.6 \%)$ of VEOIBD and 18 (6.6\%) of LO-PIBD were born of consanguineous marriage $(P=0.38)$.

\section{Delay in Diagnosis}

Diagnostic delay ( $>6$ months) was significantly lower in VEOIBD $(40.9 \%)$ than in LO-PIBD $(78.8 \%)(P<0.001)$. The median diagnostic delay in VEOIBD and LO-PIBD were 6 months (IQR, 2-30 months) and 12 months (IQR, 7-24 months), respectively $(P=0.034)$. On further analysis, there was no significant difference in diagnostic delays between UC (median, 18 months; IQR, 3-29 months) and CD (median, 20 months; IQR, 6-30 months) in each group $(P=0.30)$.

\section{Risk Factors for Diagnostic Delay}

Logistic regression analyses of risk factors for diagnostic delays ( $>50$ th percentile) in the diagnosis of CD and UC are depicted in Table 3. In CD, ileal disease was a risk factor for diagnostic delay ( $>50$ th percentile) $(P=0.03$; odds ratio [OR], 2.16). In UC, none of the factors were predictive except age $>10$ years (which nearly achieved significance: $P=0.06 ; \mathrm{OR}, 0.21$ ).

\section{ATT Prior to Diagnosis}

A total of 31 children (17.7\%; 2 in VEOIBD, 29 in LO-PIBD) of pediatric CD received ATT prior to diagnosis. Pediatric UC patients of 1.8\% (2/113) received ATT. Empirical ATT use was lower in VEOIBD compared to LO-PIBD, but not statistically significant $(P=0.49)$.

\section{Disease Extent and Severity}

Fig. $1 \mathrm{~B}$ and $\mathrm{C}$ show the extent of disease in UC and CD in VEOIBD and LO-PIBD patients.

\section{1) Ulcerative Colitis}

A higher proportion of patients with VEOIBD had extensive colitis compared to LO-PIBD (62.5\% vs. $50.5 \%)$. However, the difference was not statistically significant $(P=0.68)$ (Fig. 1B). Similarly, the severity of disease was significantly higher for VEOIBD than LO-PIBD (Table 2). The median PUCAI score for VEOIBD was 60 (range, 45-80). The severity of disease according to the PUCAI score was moderate and severe in $37.5 \%$ and $62.5 \%$, respectively with no mild cases. This was significantly higher than in LO-PIBD where $35 \%$ had mild disease (Table 2). 
Table 2. Disease Characteristics of VEOIBD ( $<6$ Years) and LO-PIBD (6-17 Years) Separately in Ulcerative Colitis and Crohn's Disease

\begin{tabular}{|c|c|c|c|c|c|c|}
\hline \multirow{2}{*}{ Characteristics } & \multicolumn{2}{|c|}{ UC, No. (\%) } & \multirow{2}{*}{$P$-value } & \multicolumn{2}{|c|}{ CD, No. (\%) } & \multirow{2}{*}{$P$-value } \\
\hline & VEOIBD $(n=8)$ & LO-PIBD $(n=105)$ & & VEOIBD $(n=13)$ & LO-PIBD $(n=162)$ & \\
\hline Age of onset (yr) & & & 0.170 & & & $<0.001$ \\
\hline Mean \pm SD & $2.50 \pm 1.41$ & $13.66 \pm 4.08$ & & $3.55 \pm 1.44$ & $13.51 \pm 3.10$ & \\
\hline Median (IQR) & $2.5(1-4)$ & $15(13-17)$ & & $4(2-5)$ & $14(11.7-16)$ & \\
\hline Diagnostic delay (mo) & & & 0.140 & & & 0.530 \\
\hline Mean (range) & $6.2(1-12)$ & $20.6(1-120)$ & & $19.0(1-60)$ & $24.0(1-120)$ & \\
\hline SD & 3.3 & 27.4 & & 20.4 & 28.3 & \\
\hline Median (IQR) & $6(3-9)$ & $11(6-24)$ & & $19(3.25-36)$ & $12(7-24)$ & \\
\hline \multicolumn{7}{|l|}{ Clinical features } \\
\hline Abdominal pain & $5(62.5)$ & $59(56.2)$ & 0.510 & $6(46.2)$ & $121(74.7)$ & 0.470 \\
\hline Diarrhea & 7 (87.5) & $82(78.1)$ & 0.680 & $10(76.9)$ & 102 (62.9) & 0.550 \\
\hline Blood in stool & $7(87.5)$ & 88 (83.8) & 0.630 & 7 (53.8) & $51(31.5)$ & 0.130 \\
\hline Mucus in stool & $6(75.0)$ & $53(50.5)$ & 0.270 & $4(30.8)$ & 35 (21.6) & 0.490 \\
\hline Weight loss & $3(37.5)$ & $49(46.7)$ & 0.720 & $5(38.5)$ & $86(53.1)$ & 0.390 \\
\hline Fever & $3(37.5)$ & $22(20.9)$ & 0.370 & $5(38.5)$ & 55 (33.9) & 0.770 \\
\hline EIMs & $2(25.0)$ & $27(25.7)$ & 0.660 & $3(23.1)$ & 32 (19.8) & 0.500 \\
\hline Family history of IBD & $1(12.5)$ & $10(9.5)$ & 0.570 & $2(15.4)$ & $15(9.2)$ & 0.600 \\
\hline History of consanguinity & $2(25.0)$ & $6(5.7)$ & 0.090 & $1(7.7)$ & $12(7.4)$ & 0.640 \\
\hline Extent of disease (UC) & & & 0.680 & & & \\
\hline Proctitis (E1) & $2(25.0)$ & $12(11.4)$ & & & & \\
\hline Procto-sigmoiditis (E2) & $1(12.5)$ & 16 (15.2) & & & & \\
\hline Upto hepatic flexure (E3) & 0 & $24(22.9)$ & & & & \\
\hline Pancolitis & $5(62.5)$ & $53(50.5)$ & & & & \\
\hline Extent of disease (CD) & & & & & & 0.310 \\
\hline L1 (ileal) & & & & $2(15.4)$ & $53(32.9)$ & \\
\hline L2 (colonic) & & & & $4(30.8)$ & $61(37.7)$ & \\
\hline L3 (ileo-colonic) & & & & $6(46.1)$ & $40(24.7)$ & \\
\hline$L 4 a+L 4 b^{a}$ & & & & $1(7.7)$ & $8(5.0)$ & \\
\hline Disease behavior (CD) & & & & & & 0.660 \\
\hline Inflammatory (B1) & & & & $9(69.2)$ & $91(56.2)$ & \\
\hline Stricturing (B2) & & & & $3(23.1)$ & $54(33.3)$ & \\
\hline Fistulizing (B3) & & & & $1(7.7)$ & $17(10.5)$ & \\
\hline Perianal (p) & & & & $1(7.7)$ & $12(7.4)$ & 0.968 \\
\hline Disease severity ${ }^{b}$ & & & 0.003 & & & $<0.001$ \\
\hline Mild disease & 0 & $37(35.2)$ & & $1(7.7)$ & $53(32.7)$ & \\
\hline Moderate disease & $3(37.5)$ & $55(52.4)$ & & $2(15.4)$ & 97 (59.9) & \\
\hline Severe disease & $5(62.5)$ & $13(12.3)$ & & $10(76.9)$ & $25(15.4)$ & \\
\hline \multicolumn{7}{|l|}{ Treatment history } \\
\hline 5-ASA & $8(100.0)$ & 105 (100.0) & 1.000 & $11(84.6)$ & 151 (93.2) & 0.250 \\
\hline Steroids & $6(75.0)$ & $58(55.2)$ & 0.460 & $10(76.9)$ & $112(69.1)$ & 0.600 \\
\hline Immunomodulators & $4(50.0)$ & 44 (41.9) & 0.720 & $8(61.5)$ & 96 (59.2) & 0.760 \\
\hline Biologics & $1(12.5)$ & $6(5.7)$ & 0.410 & $1(7.7)$ & $9(5.5)$ & 0.510 \\
\hline Surgery & $1(12.5)$ & $2(1.9)$ & 0.190 & $1(7.7)$ & 28 (17.2) & 0.480 \\
\hline$A T$ & 0 & $2(1.9)$ & 1.000 & $2(15.4)$ & 27 (16.6) & 0.630 \\
\hline
\end{tabular}

VEOIBD is $0+1$ and LO-PIBD is $3+5$.

'UC was classified as Pediatric Ulcerative Colitis Activity Index and CD as Pediatric Crohn's Disease Activity Index.

IBD, inflammatory bowel disease; VEOIBD, very early onset IBD; LO-PIBD, later onset pediatric IBD; UC, ulcerative colitis; CD, Crohn's disease; SD, standard deviation; IQR, interquartile range; EIM, extraintestinal manifestation; ASA, aminosalicylic acid; $A \Pi$, anti-tubercular therapy. 
Table 3. Analysis of Risk Factors Associated with Diagnostic Delay (>50th Percentile)

\begin{tabular}{lccc}
\hline Risk factor & OR & $95 \%$ Cl & $P$-value \\
\hline Ulcerative colitis & & & \\
Sex (male or female) & 1.59 & $0.68-3.75$ & 0.28 \\
Onset of symptoms ( $>10$ or $\leq 10 \mathrm{yr}$ ) & 0.21 & $0.04-1.07$ & 0.06 \\
AT use (yes or no) & 0.72 & $0.04-12.76$ & 0.82 \\
Disease location (pancolitis or others) & 0.86 & $0.24-3.17$ & 0.82 \\
Family history (yes or no) & 1.91 & $0.53-2.68$ & 0.67 \\
Crohn's disease & & & \\
Sex (male or female) & 0.72 & $0.37-1.41$ & 0.34 \\
Onset of symptoms (> 10 or $\leq 10$ yr) & 1.78 & $0.69-4.59$ & 0.23 \\
ATT use (yes or no) & 0.63 & $0.26-1.52$ & 0.30 \\
Disease location (ileal or colonic) & 2.16 & $1.08-4.32$ & $0.03^{\mathrm{a}}$ \\
Family history (yes or no) & 1.94 & $0.59-6.23$ & 0.27 \\
\hline
\end{tabular}

${ }^{a}$ Statistically significant, $P<0.05$.

$\mathrm{OR}$, odds ratio; $\mathrm{Cl}$, confidence interval; $\mathrm{AT}$, anti-tubercular therapy.

\section{2) Crohn's Disease}

Ileo-colonic involvement (L3) was more common in VEOIBD group than LO-PIBD patients. Ileal and colonic diseases were more common in LO-PIBD (Fig. 1C). However, none of the differences were statistically significant (0.31). Inflammatory disease phenotype (B1) was more common in both VEOIBD and LO-PIBD group in CD. Stricturing (B2) and fistulizing (B3) diseases were more common in LO-PIBD, but no statistically significant difference was noted $(P=0.66)$. Perianal was found in similar proportion of patients in both subgroups $(7.7 \%$ in VEOIBD group and $7.4 \%$ in LO-PIBD group) $(P=0.97)$ (Fig. 1D). The median PCDAI score for VEOIBD was 47.5. Mild, moderate, and severe disease were found in $7.5 \%, 15.4 \%$, and $76.9 \%$ of children, respectively. This was significantly higher than the LO-PIBD group (Table 2).

\section{Infantile Onset IBD $(<2$ Years $)$}

IOIBD ( $<2$ years at onset of symptoms) was noted in 8 children (2.7\%) of PIBD. Of these, 3 (37.5\%) had complications with an aggressive disease course. The first child with CD was symptomatic for 1 month of age, with colonic ulcerations and extensive skin excoriations. His elder sibling had died of similar complaints at 1.5 years of age. Genetic testing in this child revealed interleukin (IL)-10 receptor mutations. He underwent a bone marrow transplant and was better at 8 months post-transplant. The second child required hemicolectomy for severe unresponsive disease at 6 years of age. The third child with UC was lost to follow-up for more than 2 years and expired at 5 years of age presumably due to septicemia. The 5 other cases with IOIBD also had a severe disease course with multiple relapses needing steroids.

\section{Treatment Received}

There was no significant difference in the use of biologics, steroids, 5-aminosalicylic acid (5-ASA), and immune modulators (azathioprine/methotrexate) between VEOIBD and LO-PIBD (in total as well as in subgroups of UC and CD there was no significant difference) (Table 2).

All patients with UC received 5-ASA. Sixty-four out of 113 patients (56.6\%) required a course of steroids. Steroid dependent and refractory UC were put on immunomodulators to maintain remission $(42.4 \%, 48 / 113)$. Eleven out of 113 patients with UC $(9.7 \%)$ had frequent relapsing course of disease ( $>3$ relapses a year) and 3 patients had acute severe colitis requiring surgery (2.65\%). Fifteen out of 175 CD patients (8.5\%) had $>3$ relapses in a year. Steroid and azathioprine requirements were higher in CD but biologic usage was still low $(5.7 \%, 10 / 175)$ (Table 2). Thirty-seven out of $175 \mathrm{CD}$ patients (21.1\%) received partial enteral nutrition (PEN) (approximately half of the daily calorie requirement) in addition to other therapy.

Surgery was required in 3 of $22(13.6 \%)$ of VEOIBD and 29 of 270 (10.7\%) LO-PIBD children. These included proctocolectomy (1), sub-total colectomy (3), right hemicolectomy (1), segmental small bowel resection (12 ileal, 6 jejunal) and ileocecal resection (5). Fistulectomy and examination under anesthesia with seton placement was done in 4 patients. $16.5 \%$ (29/175) patients with CD and 2.4\% (3/114) patients with UC underwent surgery. Logistic regression analysis was done to assess for risk factors of surgery-none of the risk factors significantly predicted surgery (Supplementary Table 1). There were 4 teenage smokers in our pediatric cohort (3 CD and 1 UC).

\section{DISCUSSION}

This is a retrospective analysis of prospectively maintained database of a large number of PIBD patients. This is the first study from India that has highlighted the disease characteristics and treatment patterns of PIBD particularly VEOIBD and IOIBD and compared the differences with later onset disease (6-17 years). Severity of disease was significantly higher in VEOIBD (in both UC and CD subgroups) compared to later onset disease ( $P=0.003$ and $P<0.001$, respectively) consistent with earlier studies. ${ }^{27,28}$ 
A family history of IBD was found in $14 \%$ patients with VEOIBD compared to $9 \%$ in later onset disease. The entire cohort from our registry has a positive family history in only $4 \%$ (CD of $4.13 \%$ and UC of $4.34 \%$ ) which we have published recently. ${ }^{29}$ This has been reported in many other studies that familial IBD is less common in Asia than the West. ${ }^{30,31}$ This clearly demonstrates that a genetic predisposition to IBD correlates with the onset of disease being highest in the IOIBD and VEOIBD.

We compared our data with that of other studies both in Asia and the West (Supplementary Tables 2, 3). The large Western population-based Epidemiology of inflammatory bowel diseases: new insights from a French population-based registry (EPIMAD) (1,412 PIBD patients) was used for comparison (Supplementary Table 2) ${ }^{32}$ Male preponderance was more evident ( $82 \%$ vs. $52 \%, P=0.02$ ). The VEOIBD disease character otherwise was similar to our study. Interestingly the LO-PIBD group in our study had significant differences compared to EPIMAD study. A higher number of patients had a diagnostic delay $>6$ months $(78.8 \%$ vs. $30 \%, P=0.004)$ and the incidence of familial IBD ( $9.2 \%$ vs. $15 \%, P=0.009)$ was significantly lower. The Indian PIBD appears to be more severe with a higher incidence of stricturing and penetrating disease (B2 and B3) in $\mathrm{CD}$ and pan colitis (E4) in UC compared to EPIMAD cohort. ${ }^{31}$

We also compared our data with other PIBD cohorts from Asia and Europe (Supplementary Table 3) ${ }^{6,7,16,17,32}$ We noted a high incidence of ileal CD (31.4\%) though colonic disease was most common (37.1\%). ${ }^{10}$ Another Indian study by Sonavane et al. ${ }^{17}$ similarly showed that the most common phenotype was ileal (37.5\%). Perianal disease and upper gastrointestinal (L4) was less common. A recent study on PIBD from East Asia (Japan) showed higher incidence of upper gastrointestinal and perianal disease compared to European Cohort (EUROKIDS). ${ }^{32,33}$ The incidence of structuring and fistulizing disease was significantly higher than other studies. The rates of surgery in CD (16.5\%) comparable to other Asian study (South Korea, 22.7\%). ${ }^{34,35}$ In UC, although pan colitis was the most common phenotype, colectomy rates were lower (2.7\%) supporting the notion that UC is a milder disease in Asian countries with less incidence of acute severe colitis and colectomy (Supplementary Table 3). ${ }^{13,16,36,37}$ Compared to another questionnaire-based survey from India by Sathiyasekaran et al., ${ }^{16}$ the proportion of VEOI$\mathrm{BD}$, disease location and proportion of perianal CD were different. Such differences could be due to variation in methods of data collection and study settings. ${ }^{22}$ However, results appear more consistent with a recent study from India by Sonavane et al. ${ }^{17}$
We found that a diagnostic delay $>6$ months was lesser in VEOIBD compared to later onset disease ( $40.9 \%$ vs. $78.8 \%$, $P<0.001)$ disease perhaps because of more severe disease at presentation prompting early referral in VEOIBD. Another plausible explanation could be the use of diapers in infants and hence easier observation of their stools. Ileal disease (L1) was predictive of longer diagnostic delay ( $>50$ th percentile) in $\mathrm{CD}^{38}$ This is in sharp contrast to the EPIMAD study where diagnostic delay $>6$ months were seen in only $27 \%$ and $30 \%$ patients respectively in VEOIBD and LO-PIBD. ${ }^{32}$ The disproportionately longer delay in diagnosis could be attributable to poor physician awareness of pediatric IBD in India and high percentage of ileal (31.4\%) disease in our study which is a known risk factor for diagnostic delay in pediatric $\mathrm{CD}^{39}$

The other contributory factors for diagnostic delay include the infectious mimics of IBD and over the counter antibiotics (like fluoroquinolone and metronidazole) are easily available and is often the first line of treatment. The high prevalence of TB which is often symptomatically, endoscopically and histopathologically difficult to differentiate from ileo-colonic CD is another diagnostic dilemma which results in empirical use of ATT. ${ }^{39-42}$ Almost one-fifth of children with CD in our cohort had received ATT prior to diagnosis adding to diagnostic delay. We have earlier reported that empirical use of ATT was the single largest contributor for diagnostic delay in an adult CD cohort of more than 700 patients which resulted in higher long term stenotic complications and need for surgery. ${ }^{43}$ India is endemic for $\mathrm{TB}$ and there is limited awareness about CD. Hence at the community level, patients are initiated on ATT and $\mathrm{CD}$ is diagnosed only if there is no response leading to inordinate delays in diagnosis.

We also compared data between the adult and PIBD cohort in our registry (Supplementary Table 4). Familial IBD was more common in PIBD cohort. Higher incidence of ileal disease (L1), stricturing phenotype (B2) and perianal disease was found in pediatric CD cohort. UC in PIBD cohort was more extensive. Steroid, immunomodulator, and biologic usage was also higher in PIBD group. However, the diagnostic delay was significantly higher $(P<0.001)$ in adults with CD $($ mean \pm standard deviation [SD], $42.2 \pm 46.1$ months) compared to pediatric CD (mean \pm SD, $23.56 \pm 27.76$ months). ${ }^{22}$ This could be attributable to the higher usage of empirical ATT in the adult cohort.

IOIBD was seen in 8 children ( $2.7 \%$ of PIBD). Three had refractory disease requiring surgery. IL-10 receptor mutation was detected in 1 child who later underwent allogenic hematopoietic stem cell transplantation. This is very much in con- 
cordance with other studies from across the globe suggesting that IOIBD is a distinct and the most difficult to treat subset of pediatric IBD. ${ }^{44}$ Monogenic defects like IL-10/IL-10R mutations are not uncommon in this subgroup. ${ }^{45,46}$ However, mutational defects often go undetected due to unavailability of required facilities and financial constraints in emerging economies like India. Only 1 of our VEOIBD patients was tested for receptor mutations.

The rate of steroid and biologic usage did not differ between the VEOIBD and LO-PIBD group. Although the disease severity was high and complicated and extensive disease phenotypes were common in our study, biologic usage was limited in our study. The risk of opportunistic infections, high prevalence of latent TB and of course the high cost with limited or no insurance coverage precludes biological therapy in the resource limited country settings like India. ${ }^{47}$ Exclusive enteral nutrition was not used due to poor acceptability and compliance in addition to cost concerns. However, PEN was used as an adjunct to other therapies. PEN in CD is known to be associated with an extended period of disease remission. ${ }^{48}$

We acknowledge certain limitations including, the relatively small number of children in VEOIBD group and inability to perform mutation analysis in a large fraction of children. We did not assess all the PIBD patients routinely for growth failure- so we could not assess the extent of growth failure in various groups which is important aspect of PIBD specially in CD. ${ }^{49}$ It was also a single tertiary care center analysis with the possibility of overrepresentation of severe disease. Comparisons with the Western and other Asian data are at best tentative.

This is the first PIBD cohort from India highlighting the differences between VEOIBD and LO-PIBD in children. VEOIBD though uncommon was associated with more severe disease and poorer outcomes compared to later onset disease. VEOIBD in India was phenotypically similar to the West although later onset (6-17 years) disease phenotype differed. Large multicenter studies based on prospective databases are required to confirm whether PIBD in India is phenotypically different. We found that diagnostic delays were common and most children received multiple courses of antibiotics before confirmation of diagnosis. This would have significant long term prognostic implications. This study highlights the urgent need for increasing the awareness on the prevalence of IBD in the pediatric population amongst primary care physicians to ensure early diagnosis and timely treatment of this chronic disease.

\section{ADDITIONAL INFORMATION}

\section{Funding Source}

The authors received no financial support for the research, authorship, and/or publication of this article.

\section{Conflict of Interest}

No potential conflict of interest relevant to this article was reported.

\section{Author Contribution}

Conceptualization: Banerjee R. Data curation: Pal P, Shava U, Ganesh G. Formal analysis: Pal P. Investigation: Pal P. Methodology: Banerjee R. Project administration: Banerjee R, Reddy DN. Resources: Banerjee R. Supervision: Banerjee R. Visualization: Banerjee R, Pal P. Writing - original draft: Banerjee R, Pal P, Nabi Z, Shava U, Ganesh G. Writing - review \& editing: all authors. Approval of final manuscript: all authors.

\section{ORCID}

Banerjee R https://orcid.org/0000-0002-3753-4933

Pal P https://orcid.org/0000-0002-7090-9004

Nabi Z https://orcid.org/0000-0003-2713-4781

Shava U https://orcid.org/0000-0002-2233-0161

Ganesh G https://orcid.org/0000-0002-3909-4206

Reddy DN https://orcid.org/0000-0001-7540-0496

\section{Supplementary Material}

Supplementary materials are available at the Intestinal Research website (https://www.irjournal.org).

\section{REFERENCES}

1. Conrad MA, Rosh JR. Pediatric inflammatory bowel disease. Pediatr Clin North Am 2017;64:577-591.

2. Benchimol EI, Fortinsky KJ, Gozdyra P, Van den Heuvel M, Van Limbergen J, Griffiths AM. Epidemiology of pediatric inflammatory bowel disease: a systematic review of international trends. Inflamm Bowel Dis 2011;17:423-439.

3. Roberts SE, Thorne K, Thapar N, et al. A systematic review and meta-analysis of paediatric inflammatory bowel disease incidence and prevalence across Europe. J Crohns Colitis 2020; 14:1119-1148.

4. Huang JG, Aw MM. Pediatric inflammatory bowel disease in Asia: epidemiology and natural history. Pediatr Neonatol 2020; 61:263-271. 
5. Ong C, Aw MM, Liwanag MJ, Quak SH, Phua KB. Rapid rise in the incidence and clinical characteristics of pediatric inflammatory bowel disease in a South-East Asian cohort in Singapore, 1994-2015. J Dig Dis 2018;19:395-403.

6. Shen YM, Wu JF, Chen HL, et al. Characteristics and incidences of pediatric Crohn's disease in the decades before and after 2000. Pediatr Neonatol 201 1;52:317-320.

7. Dalzell AM, Ba'Ath ME. Paediatric inflammatory bowel disease: review with a focus on practice in low- to middle-income countries. Paediatr Int Child Health 2019;39:48-58.

8. Wang XQ, Zhang Y, Xu CD, et al. Inflammatory bowel disease in Chinese children: a multicenter analysis over a decade from Shanghai. Inflamm Bowel Dis 2013;19:423-428.

9. Jung YS, Han M, Kim WH, Park S, Cheon JH. Incidence and clinical outcomes of inflammatory bowel disease in South Korea, 2011-2014: a nationwide population-based study. Dig Dis Sci 2017;62:2102-2112.

10. Ishige T, Tomomasa T, Hatori R, et al. Temporal trend of pediatric inflammatory bowel disease: analysis of national registry data 2004 to 2013 in Japan. J Pediatr Gastroenterol Nutr 2017; 65:e80-e82.

11. Hong SN, Kim HJ, Kim KH, Han SJ, Ahn IM, Ahn HS. Risk of incident Mycobacterium tuberculosis infection in patients with inflammatory bowel disease: a nationwide populationbased study in South Korea. Aliment Pharmacol Ther 2017;45: 253-263.

12. Banerjee R, Pal P, Nugent Z, et al. IBD in India: similar phenotype but different demographics than the west. J Clin Gastroenterol 2020;54:725-732.

13. Wang XQ, Xiao Y, Xu X, et al. Study of disease phenotype and its association with prognosis of paediatric inflammatory bowel disease in China. BMC Pediatr 2018;18:229.

14. Lee YM, Fock K, See SJ, Ng TM, Khor C, Teo EK. Racial differences in the prevalence of ulcerative colitis and Crohn's disease in Singapore. J Gastroenterol Hepatol 2000;15:622-625.

15. Tan YM, Goh KL. Ulcerative colitis in a multiracial Asian country: racial differences and clinical presentation among Malaysian patients. World J Gastroenterol 2005;11:5859-5862.

16. Sathiyasekaran M, Bavanandam S, Sankaranarayanan S, et al. A questionnaire survey of pediatric inflammatory bowel disease in India. Indian J Gastroenterol 2014;33:543-549.

17. Sonavane AD, Sonawane P, Amarapurkar DN. Inflammatory bowel disease across the age continuum: similarity and disparity. Indian J Pediatr 2018;85:989-994.

18. Makharia GK, Ramakrishna BS, Abraham P, et al. Survey of inflammatory bowel diseases in India. Indian J Gastroenterol
2012;31:299-306.

19. Sathiyasekaran M, Raju BB, Shivbalan S, Rajarajan K. Pediatric Crohn's disease in South India. Indian Pediatr 2005;42:459463.

20. Avinash B, Dutta AK, Chacko A. Pediatric inflammatory bowel disease in South India. Indian Pediatr 2009;46:639-640.

21. Singh SK, Srivastava A, Kumari N, Poddar U, Yachha SK, Pandey CM. Differentiation between Crohn disease and intestinal tuberculosis in children. J Pediatr Gastroenterol Nutr 2018; 66:e6-e11.

22. Banerjee R, Basavaraju U, Adigopula B, Reddy N. A well-maintained inflammatory bowel disease (IBD) registry can help assess and optimise treatment for the local population: a 10year experience in an Indian IBD registry cohort. J Crohns Colitis 2016;10(Suppl 1):S469-S470.

23. Levine A, Koletzko S, Turner D, et al. ESPGHAN revised porto criteria for the diagnosis of inflammatory bowel disease in children and adolescents. J Pediatr Gastroenterol Nutr 2014; 58:795-806.

24. Levine A, Griffiths A, Markowitz J, et al. Pediatric modification of the Montreal classification for inflammatory bowel disease: the Paris classification. Inflamm Bowel Dis 2011;17:1314-1321.

25. Hyams JS, Ferry GD, Mandel FS, et al. Development and validation of a pediatric Crohn's disease activity index. J Pediatr Gastroenterol Nutr 1991;12:439-447.

26. Turner D, Otley AR, Mack D, et al. Development, validation, and evaluation of a pediatric ulcerative colitis activity index: a prospective multicenter study. Gastroenterology 2007;133 423-432.

27. Kelsen JR, Conrad MA, Dawany N, et al. The unique disease course of children with very early onset-inflammatory bowel disease. Inflamm Bowel Dis 2020;26:909-918.

28. Moon JS. Clinical aspects and treatments for pediatric inflammatory bowel disease. Intest Res 2019;17:17-23.

29. Banerjee R, Pal P, Hutfless S, Ganesh BG, Reddy DN. Familial aggregation of inflammatory bowel disease in India: prevalence, risks and impact on disease behavior. Intest Res 2019; 17:486-495.

30. Bayless TM, Tokayer AZ, Polito JM 2nd, Quaskey SA, Mellits ED, Harris ML. Crohn's disease: concordance for site and clinical type in affected family members--potential hereditary influences. Gastroenterology 1996;111:573-579.

31. Bequet E, Sarter H, Fumery M, et al. Incidence and phenotype at diagnosis of very-early-onset compared with later-onset paediatric inflammatory bowel disease: a population-based study [1988-2011]. J Crohns Colitis 2017;11:519-526. 
32. de Bie CI, Paerregaard A, Kolacek S, et al. Disease phenotype at diagnosis in pediatric Crohn's disease: 5-year analyses of the EUROKIDS Registry. Inflamm Bowel Dis 2013;19:378-385.

33. Arai K, Kunisaki R, Kakuta F, et al. Phenotypic characteristics of pediatric inflammatory bowel disease in Japan: results from a multicenter registry. Intest Res 2020;18:412-420.

34. Kim HJ, Oh SH, Kim DY, et al. Clinical characteristics and longterm outcomes of paediatric Crohn's disease: a single-centre experience. J Crohns Colitis 2017;11:157-164

35. Hong SJ, Cho SM, Choe BH, et al. Characteristics and incidence trends for pediatric inflammatory bowel disease in DaeguKyungpook province in Korea: a multi-center study. J Korean Med Sci 2018;33:e132.

36. Ng SC. Emerging trends of inflammatory bowel disease in Asia. Gastroenterol Hepatol (N Y) 2016;12:193-196.

37. Ng SC, Zeng Z, Niewiadomski O, et al. Early course of inflammatory bowel disease in a population-based inception cohort study from 8 countries in Asia and Australia. Gastroenterology 2016;150:86-95.

38. El Mouzan MI, AlSaleem BI, Hasosah MY, et al. Diagnostic delay of pediatric inflammatory bowel disease in Saudi Arabia. Saudi J Gastroenterol 2019;25:257-261.

39. Leong RW, Mitrev N, Ko Y. Hygiene hypothesis: is the evidence the same all over the world? Dig Dis 2016;34:35-42.

40. Shaw SY, Blanchard JF, Bernstein CN. Association between the use of antibiotics in the first year of life and pediatric inflammatory bowel disease. Am J Gastroenterol 2010;105:26872692
41. Hviid A, Svanström H, Frisch M. Antibiotic use and inflammatory bowel diseases in childhood. Gut 2011;60:49-54.

42. Ma JY, Tong JL, Ran ZH. Intestinal tuberculosis and Crohn's disease: challenging differential diagnosis. J Dig Dis 2016;17: 155-161.

43. Banerjee R, Pal P, Girish BG, Reddy DN. Risk factors for diagnostic delay in Crohn's disease and their impact on long-term complications: how do they differ in a tuberculosis endemic region? Aliment Pharmacol Ther 2018;47:1367-1374.

44. Kammermeier J, Dziubak R, Pescarin M, et al. Phenotypic and genotypic characterisation of inflammatory bowel disease presenting before the age of 2 years. J Crohns Colitis 2017;11: 60-69.

45. Uhlig HH, Schwerd T, Koletzko S, et al. The diagnostic approach to monogenic very early onset inflammatory bowel disease. Gastroenterology 2014;147:990-1007.

46. Zhu L, Shi T, Zhong C, Wang Y, Chang M, Liu X. IL-10 and IL10 receptor mutations in very early onset inflammatory bowel disease. Gastroenterology Res 2017;10:65-69.

47. Ng SC, Chan FK. Infections and inflammatory bowel disease: challenges in Asia. J Dig Dis 2013;14:567-573.

48. Di Caro S, Fragkos KC, Keetarut K, et al. Enteral nutrition in adult Crohn's Disease: toward a paradigm shift. Nutrients 2019;11:2222.

49. Ishige T. Growth failure in pediatric onset inflammatory bowel disease: mechanisms, epidemiology, and management. Transl Pediatr 2019;8:16-22. 
See "Very early onset inflammatory bowel disease in a South Asian country where inflammatory bowel disease is emerging: a distinct clinical phenotype from later onset disease" on page 398-407.

Supplementary Table 1. Logistic Regression Analysis of Risk Factors of Surgery

\begin{tabular}{lccc}
\hline Risk factor & $P$-value & OR & 95\% Cl \\
\hline Location of disease (ileal or colonic) & 0.78 & 0.88 & $0.36-2.16$ \\
Disease behavior (B2 or others) & 0.56 & 1.32 & $0.52-3.35$ \\
Smoking (yes or no) & 0.99 & 4.02 & $0.01-8.42$ \\
ATT (yes or no) & 0.05 & 0.38 & $0.14-1.00$ \\
Sex (male or female) & 0.79 & 1.12 & $0.48-2.63$ \\
Onset of symptoms (<10 or $>10 \mathrm{yr}$ ) & 0.34 & 0.56 & $0.17-1.82$ \\
Diagnostic delay (>1 or <1 yr) & 0.37 & 1.47 & $0.63-3.43$ \\
Family history (yes or no) & 0.22 & 3.79 & $0.46-31.58$ \\
\hline
\end{tabular}

$\mathrm{OR}$, odds ratio; $\mathrm{Cl}$, confidence interval; $\mathrm{ATT}$, anti-tubercular therapy. 
Supplementary Table 2. Comparison of Pediatric Inflammatory Bowel Disease Data with French Population-Based Registry (EPIMAD)

\begin{tabular}{|c|c|c|c|c|c|c|}
\hline \multirow{2}{*}{ Variable } & \multicolumn{3}{|c|}{ VEOIBD, \% (No./No.) } & \multicolumn{3}{|c|}{ LO-PIBD, \% (No./No.) } \\
\hline & India & EPIMAD $^{31}$ & $P$-value & India & EPIMAD $^{31}$ & $P$-value \\
\hline No. of patients (\%) & $22(7.5)$ & $42(3)$ & 0.003 & $270(92.5)$ & $1,370(97)$ & \\
\hline CD:UC:IBD-U (\%) & $59.1: 36.4: 4.5$ & $60: 33: 7$ & 0.90 & 60:39:1 & $73.5: 24.0: 2.5$ & $<0.001$ \\
\hline Male sex & $82(18 / 22)$ & $52(22 / 42)$ & 0.02 & $61(165 / 270)$ & $52(708 / 1,370)$ & 0.004 \\
\hline Diagnostic delay $>6$ mo & $40.9(9 / 22)$ & $27(11 / 42)$ & 0.23 & $78.8(213 / 270)$ & $30(407 / 1,370)$ & $<0.001$ \\
\hline Family history of IBD & $13.6(3 / 22)$ & $9.5(4 / 42)$ & 1 & $9.2(25 / 270)$ & $15(210 / 1,370)$ & 0.009 \\
\hline Diarrhea & $77.2(17 / 22)$ & 76 (32/42) & 0.92 & $68.1(184 / 270)$ & $66(899 / 1,370)$ & 0.03 \\
\hline Blood in stool & $68.2(15 / 22)$ & $81(34 / 42)$ & 0.25 & $52.6(142 / 270)$ & $46(624 / 1,370)$ & $<0.001$ \\
\hline Mucus in stool & $50(11 / 22)$ & $40(17 / 42)$ & 0.47 & $33.7(91 / 270)$ & $21(281 / 1,370)$ & $<0.001$ \\
\hline Weight loss & $36.4(8 / 22)$ & $21(9 / 42)$ & 0.19 & $50.37(136 / 270)$ & $49(670 / 1,370)$ & 0.66 \\
\hline Abdominal pain & $50(11 / 22)$ & $43(18 / 42)$ & 0.58 & $67.04(181 / 270)$ & $74(1,013 / 1,370)$ & 0.15 \\
\hline EIM & $22.7(5 / 22)$ & $17(7 / 42)$ & 0.74 & $21.8(59 / 270)$ & $17(231 / 1,370)$ & $<0.049$ \\
\hline Perianal disease & $7.69(1 / 13)$ & $8(2 / 25)$ & & $7.4(13 / 162)$ & $6(59 / 1,032)$ & \\
\hline \multicolumn{7}{|l|}{ Crohn's disease } \\
\hline Bleeding per rectum & $53.8(7 / 13)$ & $68(17 / 25)$ & 0.48 & $31.5(51 / 162)$ & $30(303 / 1,007)$ & 0.72 \\
\hline Abdominal pain & $46.2(6 / 13)$ & $48(12 / 25)$ & 0.92 & $74.7(121 / 162)$ & $74(809 / 1,007)$ & 0.09 \\
\hline Weight loss & $38.5(5 / 13)$ & $20(5 / 25)$ & 0.26 & $53.1(86 / 162)$ & $56(566 / 1,007)$ & 0.46 \\
\hline EIM & $23.1(3 / 13)$ & $20(5 / 25)$ & 0.57 & $19.8(32 / 162)$ & $21(208 / 1,007)$ & 0.79 \\
\hline Colonic disease L2 & $30.8(4 / 13)$ & $39(9 / 25)$ & 0.52 & $37.7(61 / 162)$ & $14(128 / 1,007)$ & $<0.001$ \\
\hline $\mathrm{B} 2+\mathrm{B} 3$ disease in $\mathrm{CD}$ & $30.8(4 / 13)$ & $13(3 / 25)$ & 0.20 & $43.8(71 / 162)$ & $22(208 / 1,007)$ & $<0.001$ \\
\hline \multicolumn{7}{|l|}{ Ulcerative colitis } \\
\hline Abdominal pain & $62.5(5 / 8)$ & $21(3 / 14)$ & 0.08 & $56.2(59 / 105)$ & $55(180 / 329)$ & 0.79 \\
\hline EIMs & $25(2 / 8)$ & $7(1 / 14)$ & 0.53 & $25.7(27 / 105)$ & $6(19 / 329)$ & $<0.001$ \\
\hline Proctitis (E1) & $25(2 / 8)$ & $9(1 / 11)$ & 0.32 & $11.4(12 / 105)$ & $30(92 / 303)$ & $<0.001$ \\
\hline Procto-sigmoiditis (E2) & $12.5(1 / 8)$ & $36(4 / 11)$ & & $15.2(16 / 105)$ & $26(79 / 303)$ & \\
\hline Upto hepatic flexure(E3) & $0(0 / 8)$ & $27(3 / 11)$ & & $22.9(24 / 105)$ & $10(30 / 303)$ & \\
\hline Pancolitis (E4) & $62.5(5 / 8)$ & $27(3 / 11)$ & & $50.5(53 / 105)$ & $33(102 / 303)$ & \\
\hline
\end{tabular}

EPIMAD, epidemiology of inflammatory bowel diseases: new insights from a French population-based registry; IBD, inflammatory bowel disease; $V E O \mathrm{BBD}_{1}$ very early onset IBD; LO-PIBD, later onset pediatric IBD; CD, Crohn's disease; UC, ulcerative colitis; IBD-U, IBD undifferentiated; EIM, extraintestinal manifestations; L2, colonic CD; B2, stricturing disease; B3, fistulizing $C D$. 
Supplementary Table 3. PIBD Data Comparison with Indian, Asian and Western Studies

\begin{tabular}{|c|c|c|c|c|c|}
\hline Variable & $\begin{array}{c}\text { Sathiyasekaran } \\
\text { et al. }^{16} \\
(n=221)\end{array}$ & $\begin{array}{c}\text { Sonawane } \\
\text { et al. }^{17} \\
(n=65)\end{array}$ & $\begin{array}{c}\text { AIG PIBD } \\
\text { cohort } \\
(n=292)\end{array}$ & $\begin{array}{l}\text { South Korea } a^{34,35} \\
\qquad(n=574)^{34} \\
(n=122)^{35}\end{array}$ & $\begin{array}{l}\text { EUROKIDS }^{32} \\
(n=3,461)\end{array}$ \\
\hline Disease phenotype, CD/UC/IBD-U & $55.2 / 42.1 / 2.7$ & $63 / 37 / 0$ & $60 / 39 / 1$ & $80.3 / 19.7 / 0$ & $60.2 / 32.1 / 7.7$ \\
\hline CD:UC & $1.38: 1$ & $1.7: 1$ & 1.55:1 & 4.08:1 & $1.87: 1$ \\
\hline Male sex, UC/CD & $54.8 / 47.5$ & $75.0 / 75.0$ & $63.7 / 62.0$ & $45.8 / 60.6$ & $50.9 / 60.1$ \\
\hline Mean age at diagnosis, UC/CD & $10.2 / 11$ & NA/NA & $13.6 / 12.7$ & $12.9 / 13.7$ & $12.9 / 13.3$ \\
\hline VEOIBD & 15.8 & NA & 7.5 & NA & NA \\
\hline \multicolumn{6}{|l|}{$C D$} \\
\hline VEOIBD $(<6 \mathrm{yr})$ & 15.8 & NA & 7.5 & NA & NA \\
\hline Age $<10$ yr (A1a) & 32.8 & NA & 17.1 & 2.4 & 20.0 \\
\hline Age $10-17$ yr (A1b) & 45.9 & NA & 82.9 & 73.7 & 80.0 \\
\hline \multicolumn{6}{|l|}{ Disease location in $C D$} \\
\hline L1 (ileal) & 8.2 & 37.5 & 31.4 & 10.7 & 16.0 \\
\hline L2 (colonic) & 12.3 & 29.1 & 37.1 & 8.2 & 27.0 \\
\hline L3 (ileocolonic) & 72.9 & 33.3 & 26.2 & 79.3 & 53.0 \\
\hline L4 (upper GI) & 11.5 & 0 & 5.1 & 25.8 & 49.5 \\
\hline B1 (inflammatory) & 73.0 & 79.1 & 57.1 & 88.9 & 82.0 \\
\hline B2 (stricturing) & 6.5 & 8.3 & 32.5 & 9.4 & 12.0 \\
\hline B3 (fistulizing) & - & 12.5 & 10.2 & 1.6 & 5.0 \\
\hline Perianal disease & 18.0 & NA & 7.4 & 47.1 & 9.0 \\
\hline Surgery & 8.2 & 8.3 & 16.5 & 22.7 & NA \\
\hline \multicolumn{6}{|l|}{ UC } \\
\hline $\operatorname{VEOIBD}(<6 \mathrm{yr})$ & 15.8 & NA & 7.1 & NA & NA \\
\hline Age < 10 yr (A1a) & 42.0 & NA & 15.0 & 8.3 & - \\
\hline Age $10-17$ yr (A1b) & 58.0 & NA & 85.0 & 91.7 & - \\
\hline \multicolumn{6}{|l|}{ Disease location in UC } \\
\hline Proctitis & 3.2 & 2.4 & 12.4 & 20.8 & 5.0 \\
\hline Upto splenic flexure & 25.8 & 24.4 & 15.0 & 12.5 & 18.0 \\
\hline Upto hepatic flexure & NA & NA & 21.2 & 8.3 & 9.0 \\
\hline Pancolitis & 70.9 & 73.1 & 51.3 & 58.4 & 69.0 \\
\hline Surgery & 4.3 & 7.3 & 2.7 & NA & $9.0^{4}$ \\
\hline
\end{tabular}

Value are presented as percent.

IBD, inflammatory bowel disease; PIBD, pediatric IBD; AIG, Asian Institute of Gastroenterology; EUROKIDS, European multicenter study of children with new-onset IBD; CD, Crohn's disease; UC, ulcerative colitis; IBD-U, IBD undifferentiated; VEOIBD, very early onset IBD; GI, gastrointestinal; NA, not available. 
Supplementary Table 4. Comparison of Adult and Pediatric Cohort in The Inflammatory Bowel Disease Registry

\begin{tabular}{|c|c|c|c|c|}
\hline Variable & PIBD cohort CD & Adult cohort $C D^{22}$ & PIBD cohort UC & Adult cohort $U^{22}$ \\
\hline Male sex & 62.8 & 61.2 & 63.7 & 61.4 \\
\hline Age at diagnosis (yr) & $12.80 \pm 3.89$ & $34.00 \pm 12.80$ & $13.70 \pm 3.91$ & $38.20 \pm 12.70$ \\
\hline Diagnostic delay (mo) & $23.56 \pm 27.76$ & $42.2 \pm 46.1$ & $19.37 \pm 26.76$ & $37.20 \pm 43.80$ \\
\hline Smoking & 1.7 & 7.16 & 0.8 & 7.63 \\
\hline History of AT & 17.7 & 26.09 & 1.7 & 1.21 \\
\hline Family history & 8.5 & 4.13 & 9.7 & 4.34 \\
\hline \multicolumn{5}{|l|}{ Disease location in CD } \\
\hline L1 (ileal) & 31.4 & 27.3 & & \\
\hline L2 (colonic) & 37.1 & 24.3 & & \\
\hline L3 (ileocolonic) & 26.3 & 40.9 & & \\
\hline Isolated L4 (upper GI involvement) & 5.1 & 5.0 & & \\
\hline B1 (inflammatory) & 57.1 & 67.8 & & \\
\hline B2 (stricturing) & 31.4 & 22.9 & & \\
\hline B3 (fistulizing) & 10.2 & 9.4 & & \\
\hline Perianal disease & 7.4 & 2.5 & & \\
\hline \multicolumn{5}{|l|}{ Disease location in UC } \\
\hline Proctitis & & & 12.4 & 18.7 \\
\hline Proctosigmoiditis & & & 15.0 & 51.0 \\
\hline Beyond splenic flexure & & & 72.6 & 30.3 \\
\hline \multicolumn{5}{|l|}{ Treatment given $(\mathrm{UC}+\mathrm{CD})$} \\
\hline Steroids & 69.7 & 39.7 & 56.6 & 39.5 \\
\hline Immunomodulators & 59.4 & 45.9 & 38.9 & 34.6 \\
\hline Biologics & 5.7 & 5.7 & 6.2 & 1.8 \\
\hline
\end{tabular}

Value are presented as percent or mean \pm standard deviation.

IBD, inflammatory bowel disease; PIBD, pediatric IBD; CD, Crohn's disease; UC, ulcerative colitis; AT, anti-tubercular therapy; GI, gastrointestinal. 